\title{
Therapeutic neovascularization by autologous transplantation with expanded endothelial progenitor cells from peripheral blood into ischemic hind limbs ${ }^{1}$
}

\author{
Chun-ling FAN, Ping-jin GAO, Zai-qian CHE, Jian-jun LIU, Jian WEI, Ding-liang ZHU² \\ Shanghai Key Laboratory of Vascular Biology, Shanghai Institute of Hypertension, Ruijin Hospital, Shanghai Second Medical University and \\ National Key Laboratory for Medical Genomics, Health Science Center, Shanghai Institute for Biological Sciences, Chinese Academy \\ of Sciences, Shanghai 200025, China
}

\section{Key words}

vascular endothelium; hindlimb; ischemia; physiologic neovascularization; stem cell transplantation

${ }^{1}$ Project supported by grants from the National Key Program of Basic Research (No G2000056904), from the Shanghai Science and Technology Committee (No 1DJ14005), and from the Shanghai Higher Education Bureau (№ 02BQ22).

${ }^{2}$ Correspondence to Prof Ding-liang ZHU.

Phn 86-21-6437-0045, ext 610901.

Fax 86-21-5465-4498.

E-mail zhudingliang@sibs.ac.cn

Received 2005-01-27

Accepted 2005-04-27

doi: $10.1111 / \mathrm{j} .1745-7254.2005 .00168 . \mathrm{x}$

\begin{abstract}
Aim: To investigate the hypothesis that transplantation with expanded autologous endothelial progenitor cells (EPC) could enhance neovascularization. Methods: Peripheral blood mononuclear cells (PB-MNC) isolated from New Zealand White rabbits were cultured in vitro. At $\mathrm{d} 7$, the adherent cells were collected for autologous transplantation. Rabbits with severe unilateral hind limb ischemia were randomly assigned to receive phosphate-buffered saline or expanded EPC in phosphate-buffered saline, administered by intramuscular injection in 6 sites of the ischemic thigh at postoperative $\mathrm{d} 7$. Neovascularization was monitored by using the calf blood pressure ratio to indicate tissue perfusion, digital subtraction angiography to identify collateral vessel development and histological analysis of capillary density in the ischemic limb at d 35 after surgery. Results: Autologous EPC transplantation produced significant amelioration in ischemic hind limbs, as indicated by a greater calf blood pressure ratio $(0.52 \pm 0.04 v s 0.42 \pm 0.05, P<0.01)$, angiographic score $(1.44 \pm 0.06$ vs $0.98 \pm 0.08, P<0.01)$ and capillary density in muscle $\left(195.2 \pm 5.4 / \mathrm{mm}^{2}\right.$ vs $\left.169.4 \pm 6.4 / \mathrm{mm}^{2}, P<0.05\right)$, than controls. Conclusion: Transplantation of autologous expanded EPC can promote neovascularization in ischemic hindlimbs.
\end{abstract}

\section{Introduction}

The maintenance of constant tissue perfusion is necessary for physiological organ function. Under certain pathological conditions, however, vascular supply can be reduced to such an extent that tissue necrosis results. Various angiogenic approaches have already been widely explored to revascularize ischemic tissue in animal models of ischemia and in clinical trials ${ }^{[1-5]}$. Most interventions involve the delivery of angiogenic growth factors such as vascular endothelial growth factor, basic fibroblast growth factor genes encoding these proteins to the ischemic tissue to stimulate the growth of new vessels. In recent years, ample evidence has established that bone marrow-derived endothelial progenitor cells (EPC) are present in the systemic circulation, are augmented in response to certain cytokines and/or tissue ischemia, and home to as well as incorporate into sites of neovascularization (which is defined as postnatal vasculogenesis $)^{[6-9]}$. Experimental studies have shown that bone marrow-derived or blood-derived EPC may contribute to the regeneration of ischemic tissues and enhance the neovascularization of ischemic hind limbs or myocardium ${ }^{[10-14]}$. Therapeutic vasculogenesis in which EPC are actively involved is a new strategy for ischemic disease. Allogenetic or heterogenetic transplantation of EPC into ischemic animals has produced promising results, and thus brought attention to the possibility of using such treatments clinically.

To use EPC safely in a clinical setting for ischemic disease, autologous EPC are the first choice. Circulating EPC are at a very low level in adults, and the number is insufficient for 
transplantation unless they are enriched in some way ${ }^{[15,16]}$ The use of expanded EPC is one solution to the problem. Therefore, we investigated the feasibility of using intramuscular injections of autologous expanded endothelial progenitor cells to treat rabbits with hind limb ischemia.

\section{Materials and methods}

Hind limb ischemia model Twelve male New Zealand white rabbits (weighing $2.8-3.2 \mathrm{~kg}$ ) were used for this study. The initial surgery to induce hind limb ischemia has been described by others ${ }^{[17]}$. Briefly, the rabbits were first anesthetized with sodium pentobarbital (30 mg/kg, iv), then under sterile surgical conditions, a longitudinal incision was made on the medial thigh of one hind limb, extending from the inguinal ligament to a point just proximal to the patella. After the skin incision, the entire femoral artery and all its major branches including the inferior epigastria, deep femoral, lateral circumflex and superficial epigastria arteries were dissected free. The external iliac artery and all of the arteries listed were ligated. Finally, the femoral artery was excised from its proximal origin as a branch of the external iliac artery, to the point distally where it bifurcates into the saphenous and popliteal arteries. As a consequence, blood flow to the ischemic limb became completely dependent upon collateral vessels issuing from the internal iliac artery. The incision was closed in three layers with 3.0 silk. All rabbits were closely monitored by veterinary staff and received an antibiotic $(20 \mathrm{mg} / \mathrm{kg}$ gentamycin sulfate, im) for $3 \mathrm{~d}$. The experimental protocols were approved by the Institutional Animal Care and Use Committee of Shanghai Second Medical University, Shanghai.

Mononuclear cell isolation and culture Samples of peripheral blood (12-15 mL) from the central auricular artery of rabbits were harvested $1 \mathrm{~h}$ before surgery and anticoagulated with heparin. The isolation and culture of mononuclear cells has been described previously ${ }^{[18]}$. In brief, peripheral blood mononuclear cells (PB-MNC) were isolated by density-gradient centrifugation and resuspended in Iscove's Modified Dulbecco's Medium (IMDM, Gibco Invitrogen, Shanghai, China) supplemented with $20 \%$ fetal calf serum (FCS; CCT, Cansera Internationa, Ontario, Canada). The cells were then seeded into 48-well plates (Sumilon, Sumitomo Bakelite, Osaka City, Japan) at a density of $1 \times 10^{6}$ cells per well and cultured at $37{ }^{\circ} \mathrm{C}$ and $5 \% \mathrm{CO}_{2}$. The medium was replaced completely at $\mathrm{d} 3$ or $\mathrm{d} 4$.

To confirm EPC phenotype, the adherent cells were incubated with 1,1'-dioctadecyl-3,3,3',3'-tetramethyl-indocarbocyanine perchlorate-labeled acetylated low density lipopro-
tein(LDL)(DiI-Ac-LDL, $10 \mathrm{mg} / \mathrm{mL}$; Biomedical Technologies, Stoughton, USA) at $37{ }^{\circ} \mathrm{C}$ for $2 \mathrm{~h}$ at $\mathrm{d} 7$ in culture. Then the cells were fixed with $2 \%$ paraformaldehyde for $10 \mathrm{~min}$ and incubated with fluorescein isothiocyanate-labeled Ulex europeus agglutinin (UEA-1 lectin, $10 \mathrm{mg} / \mathrm{mL}$; Sigma, St Louis, USA) for $1 \mathrm{~h}$. The lectin binding is a marker of endothelial cell (EC) lineage and the incorporation of acLDL is a characteristic function of EC ${ }^{[13]}$. Nearly all adherent cells $(>97 \%$ ) were DiI-acLDL $(+)$ UEA 1-lectin $(+)$ as analyzed by a fluorescence-activated cell sorter (FACS).

Experimental groups and EPC transplantation After femoral artery excision, the rabbits were randomly assigned to one of 2 groups: (a) control group ( $n=6$, receiving vehicle PBS); (b) EPC group (receiving expanded EPC, $n=6$ ).

At d 7, EPC in culture were trypsinized and collected for transplantation. EPC in $0.6 \mathrm{~mL}$ of phosphate-buffered saline (PBS) were injected im into 6 different sites of the ischemic thigh skeletal muscles ( $100 \mathrm{~mL}$ to each site) with a 22 -gauge needle. The control group received $0.6 \mathrm{~mL}$ PBS. Overall, a mean of $2.11 \times 10^{6} \pm 0.51 \times 10^{6} \mathrm{EPC}$ were injected per rabbit.

To elucidate in vivo differentiation to endothelial lineage, $2 \times 10^{6}$ EPC from autologous PB-MNC were labeled with fluorescent carbocyanine 1,1'-dioctadecyl-3,3,3',3'-tetramethylindocarbocyanine perchlorate (DiI, Molecular Probes (Eugene, Oregon, USA) and were injected via a 22-gauge needle to the ischemic thigh muscles $7 \mathrm{~d}$ after surgery. Four weeks after cell transplantation, $5 \mathrm{mg}$ of UEA-1 lectin (Sigma) was infused intravenously before the rabbits were killed by an overdose of pentobarbital. Ischemic hind limb thigh muscles were embedded in OCT compound and snap-frozen for fluorescence microscopy to examine the incorporation of transplanted cells into foci of neovascularization.

Calf blood pressure ratio Calf blood pressure was measured at the posterior tibial artery before surgery and at 7, 14, 21, 28, and $35 \mathrm{~d}$ in both hind limbs using a Doppler Flowmeter (Model ALF 2100, Advance Advance Co, Tokyo, Japan) and a cuff (PC $2.5 \mathrm{~cm} \times 12 \mathrm{~cm}$, Hokanson, Bellevue, USA) connected to a pressure manometer as described by others ${ }^{[2]}$. The calf blood pressure ratio (BPR) was defined for each rabbit as the ratio of systolic pressure of the ischemic hind limb to that of the normal hind limb.

Intra-iliac arterial angiography The collateral arteries in the medial thigh region of the ischemic limb originate from the internal iliac artery ${ }^{[19,20]}$. Digital subtraction angiography (DSA; Model Advantx-LC-DLX, General Electronic) of the hind limb was performed on postoperative $d 35$ to obtain an anatomical measurement of the growth and development of the larger conduit vessels in the medial portion of the upper limb. Each animal was anesthetized, and the right 
common carotid artery was exposed. A 4-F polyethylene catheter (Cordis, Corporation, Miami Lakes, USA) was introduced into the right common carotid artery through a small cutdown and the tip of the catheter was positioned just proximal to the point where it bifurcates into the right and left common iliac arteries under fluoroscopic guidance. Immediately following an intra-arterial bolus injection of the vasodilator sodium nitroprusside ( $300 \mu \mathrm{g}$ in $1 \mathrm{~mL}$ saline), iodinated contrast media (Huaihai Pharmaceutical Company, Shanghai, China) was infused intra-arterially at a rate of $1 \mathrm{~mL} / \mathrm{s}$ for $5 \mathrm{~s}$ using an automated angiographic injector (LF, LiebelFlarsheim Co, Cincinnati, OH, USA). Perfusion of the hind limb was observed on a monitor in real time and a series of angiographic images were taken after the start of contrast media infusion.

The angiograms at $4 \mathrm{~s}$ were quantified by image analysis using Qwin software (Leica, Bensheim, Germany). In brief, the area occupied by all small visible arteries in the medial thigh, including the branches of the femoral artery and collateral vessels from the intra-iliac artery (excluding the intraand extra-iliac, femoral, saphenous and popliteal arteries) was calculated. The angiographic score was calculated by a single observer who was blinded to the treatment regimen, and the score was defined for each rabbit as the ratio of the area occupied by vessels of the ischemic thigh to that of the normal thigh.

Histological determination of capillary density Immediately after angiography, the rabbits were killed, then the hind limbs were dissected, and samples of the adductor muscle and the semimembranous muscle were removed for histological evaluation. The tissues were placed in plastic cassettes and covered with OCT compound (Tissue-Tek, Sakura Finetechnical Co, Tokyo, Japan) before being snap frozen in liquid nitrogen. Multiple frozen sections were cut (10 mm thickness) on a cryostat (CM 3050, Leica) and placed on glass slides. Tissue sections were stained for alkaline phosphatase using the indoxyl-tetrazolium method ${ }^{[21]}$ to detect capillary endothelial cells, and were counterstained with eosin. The number of capillaries was counted under a $20 \times$ objective lens using an image analysis system (Qwin, Leica) by a single observer blinded to the treatment regimen. Capillaries in 15 randomly selected fields were analyzed. Capillary density was calculated as the number of capillaries per $\mathrm{mm}^{2}$ of muscle.

Clinical assessment All rabbits were clinically evaluated for the incidence of distal limb ulcers and necrosis by macroscopic examination during the study; this was considered significant if a skin ulcer or necrosis was observed at the leg, foot or ankle.
Statistical analysis All data are reported as mean \pm SEM. Differences between control and EPC groups were analyzed by using the paired Student's $t$-test. A value of $P<0.05$ was considered statistically significant.

\section{Results}

Characterization of EPC expanded ex vivo Freshly isolated PB-MNC were round and small, but became larger with more cytoplasm on the second day. Large round adherent cells appeared from $\mathrm{d} 2-\mathrm{d} 3$, then they assumed a spindleshaped, endothelial cell-like morphology. Significant proliferation was observed from $\mathrm{d} 3$, and the adherent cells were almost convergent at $\mathrm{d} 6-\mathrm{d} 7$. The morphology of the adherent cells is shown in Figure 1A,1B; $>97 \%$ of the adherent cells had taken up acetylated LDL (red fluorescence) and had bound the lectin (green fluorescence) (Figure 1C, 1D). The percentage of EPC in freshly isolated PB-MNC was much less than that in the adherent cells in culture (data not shown).

The incubation of PB-MNC in vitro resulted in a 100- to 200-fold increase in differentiating EPC which were DiI-acLDL (+) UEA 1-lectin (+). This calculation is based on our findings that freshly isolated PB-MNC had much fewer (about $0.06 \%)$ EPC (data not shown). We were able to obtain $1.07 \times 10^{5} \pm 0.33 \times 10^{5} \mathrm{EPC} / 1 \times 10^{6} \mathrm{MNC}$ after $7 \mathrm{~d}$ of the $e x$ vivo culture, thus yielding a 100 - to 200 -fold increase in EPC number.

Effect of transplanted autologous EPC on neovascularization

Calf blood pressure ratio The systolic blood pressure of the ischemic hind limb showed a tendency to increase after surgery in both the EPC and control groups. EPC transplantation promoted the recovery of blood perfusion to the ischemic hind limb, as by the greater ischemic/normal calf blood pressure ratio than that observed in the control group. The ischemic/normal calf blood pressure ratio measured at $\mathrm{d}$ 28 and $\mathrm{d} 35$ was $0.47 \pm 0.02$ and $0.52 \pm 0.02$, respectively, for the EPC group, which are significantly higher than those of the control animals $(0.38 \pm 0.01$ and $0.42 \pm 0.03$; Figure 2$)$. Thus, EPC transplantation significantly increased the ischemic/ normal calf blood pressure ratio, suggesting augmented perfusion in the EPC-treated group compared to control rabbits.

Angiographic analysis Collateral vessel development in the medial thigh was assessed by digital subtraction angiography as described earlier. There were more collateral vessels in the EPC group compared with the control. The angiographic score at $\mathrm{d} 35$ in the EPC group was significantly higher than that of the control group (control $=0.98 \pm$ $0.08 ; \mathrm{EPC}=1.44 \pm 0.06 ; P<0.01$; Figure 3 ). 

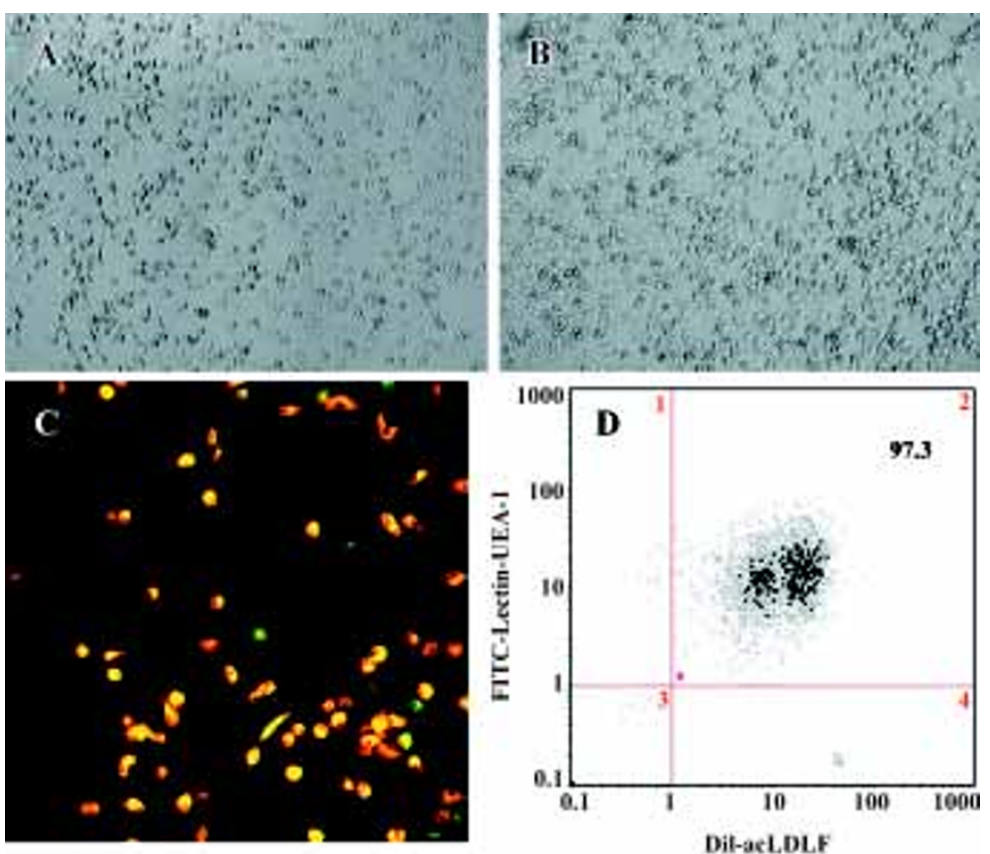

Figure 1. Characteristics of peripheral blood EPC in culture at $(\mathrm{A}) \mathrm{d} 4$ and $(\mathrm{B}) \mathrm{d} 7(\times 10)$. Cultivated EPC were incubated with DiI-acLDL (red fluorescence) and stained with lectin (green fluorescence), viewed by (C) confocal microscopy and (D) analyzed by FACS.

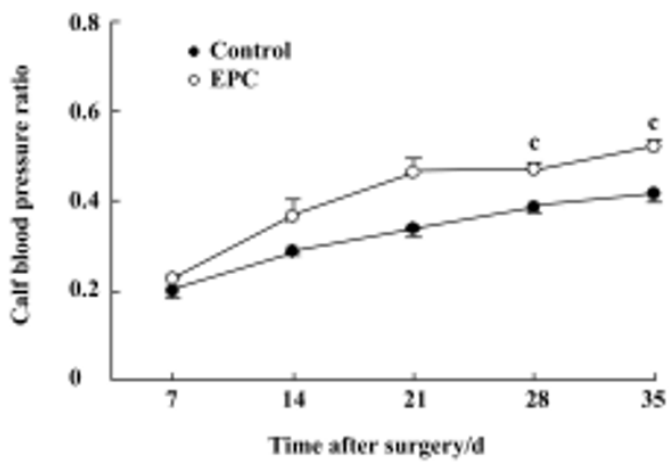

Figure 2. Time-course of the calf blood pressure ratio in the hind limbs of rabbits treated with transplanted EPC. Results are presented for animals receiving PBS (control) or transplanted EPC at d 7, 14, 21,28 , and 35 post-surgery. $n=6$. Mean \pm SEM. ${ }^{\mathrm{c}} P<0.01$ vs control.
Histological evaluation of capillary density Histological staining of alkaline phosphatase was performed to identify capillaries in ischemic muscles on d 35 after surgery. Tissue sections retrieved from the medial thigh muscles of the ischemic hind limbs showed a marked increase in capillary number in EPC-treated animals. Capillary density in the EPC group was significantly greater than that in the controls (control=169.4 $\pm 6.4 / \mathrm{mm}^{2} ; \quad E P C=195.2 \pm 5.4 / \mathrm{mm}^{2} ; P<0.05$; Figure 4).

Transplanted autologous EPC incorporate into foci of neovascularization Four weeks after autologous EPC transplantation, tissue sections from the ischemic thigh muscles were examined under fluorescence. Transplanted autologous EPC marked with DiI fluorescenced red, whereas
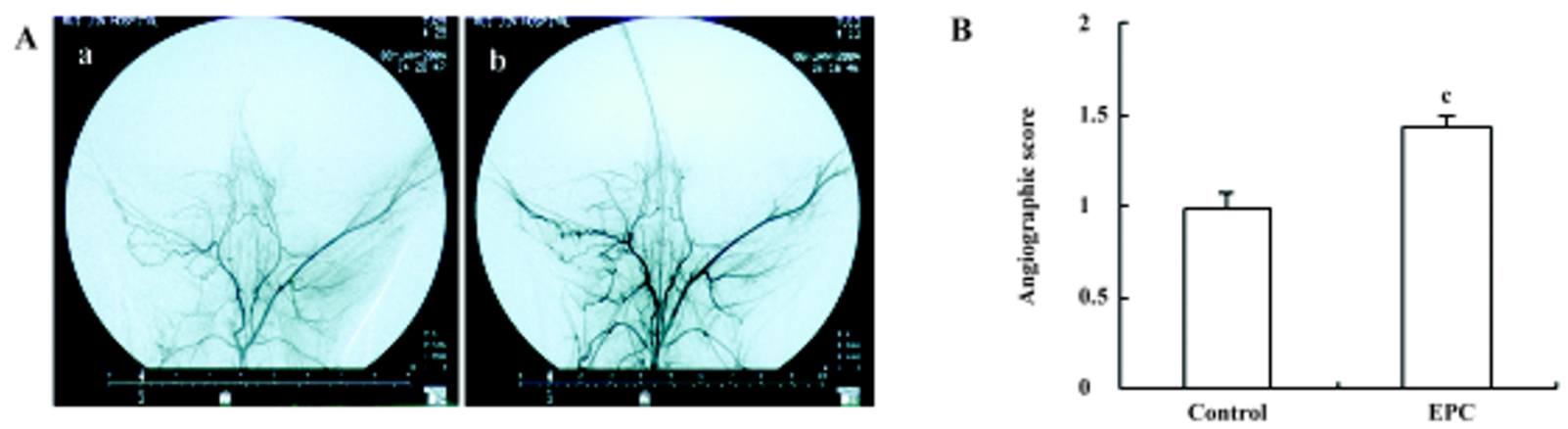

Figure 3. Effect of transplanted endothelial progenitor cells (EPC) on collateral vessel development in ischemic hind limbs of rabbits at $d 35$ post-surgery. (A) Representative angiograms of ischemic hind limbs. (a) Control rabbits receiving PBS; (b) Rabbits receiving EPC transplantation. (B) Effect of EPC transplantation on angiographic score compared with control (angiographic score is defined in the text). $n=3$. Mean \pm SEM. ${ }^{\mathrm{c}} P<0.01$ vs control. 


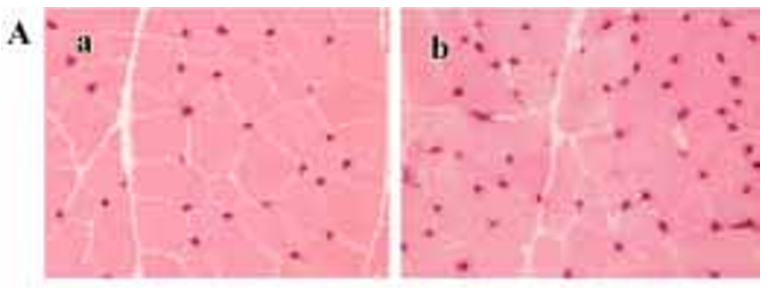

B

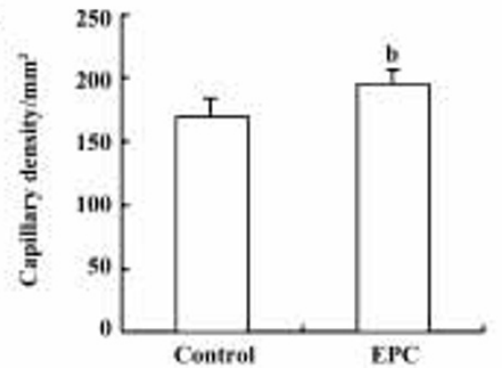

Figure 4. Histological evaluation of capillary density in ischemic hind-limb muscle on d 35 post-surgery. (A) Representative images of alkaline phosphatase-stained capillaries $(\times 20)$. (a) Control rabbits receiving PBS; (b) Rabbits receiving transplanted EPC. (B) Quantitative analysis of capillary density. $n=6$. Mean \pm SEM. ${ }^{b} P<0.05$ vs control.

the rabbit vasculature, stained by a premortem administration of UEA-1 lectin, were identified by green fluorescence in the same tissue section. DiI-labeled EPC were distributed principally in the ischemic area of the hind limb neovascularization and incorporated into tubular structures and capillaries, which is consistent with neovasculature (Figure 5). Thus, locally transplanted EPC were incorporated into the foci of neovascularization and differentiated into mature endothelial cells in the ischemic hind limbs.
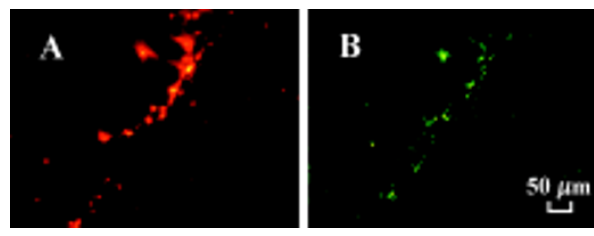

Figure 5. Histological identification of epithelial progenitor cells (EPC) incorporation in vivo. Tissue sections were retrieved from ischemic muscles. (A) Red fluorescence marks autologously transplanted DiI-labeled EPC. (B) Green fluorescence indicates UEA-1 lectin binding identifying endothelial cells $(\times 10)$.

Clinical assessment All animals were conscious within $1 \mathrm{~h}$ of the surgery, and they developed a limp in their hind limbs. Two animals from the control group developed skin ulcers at the knees of the ischemic limb. Animals from the EPC group did not have any skin ulcers or necrosis.

\section{Discussion}

The present study describes for the first time autologous transplantation with expanded EPC from peripheral blood in hind limb ischemia. We found that the transplantation of EPC was associated with a significant increase in the calf blood pressure ratio, collateral vessel development, and muscle capillary density, suggesting a beneficial effect on neovascularization.

Postnatal EPC have been implicated in the neovascularization associated with postnatal vasculogenesis. In animal experiments, allogenetic or heterogenetic transplantation of blood-derived cells containing EPC into ischemic animals have been shown to regenerate infarcted myocardium and coronary capillaries, thus limiting functional impairment after myocardial ischemia. Enhanced neovascularization by EPC was also observed in animals with hind limb ischemia.

After favorable results were found in studies of allogenetic and heterogenetic transplantations of EPC, studies were carried out to investigate the efficiency of autologous transplantation of EPC in animals and humans ${ }^{[15,16,22-28]}$ to overcome the limitations of immunorejection, but these studies did not answer the crucial questions such as cell populations for transplantation, delivery method, and therapy time for cell transplantation, which are important for efficient transplantation.

In the present study, we used expanded EPC for transplantation based on the following considerations. First, the number of EPC after expansion greatly exceeds the number of EPC in freshly isolated cells, which need mononuclear cell apheresis to enrich the EPC-containing cell population. Second, expanded EPC are pure and uncontaminated, and are exempt from the potential inflammatory response induced by other cells from a hematopoietic lineage. In the present study, EPC expanded 100- to 200-fold in number after $7 \mathrm{~d}$ of culture in vitro. Kalka et al reported a 80- to 90-fold expansion of EPC when cultured in a fibronectin-coated dish in a commercial medium (EBM-2) supplemented with a cocktail of growth factors including VEGF, bFGF, insulin-like growth factor (IGF), and epidermal growth factor (EGF) ${ }^{[13]}$. Our study used a simple medium supplemented with FCS, but which did not compromise the expansion potential. The culture conditions used in the present study are much more economical and accessible than those used by Kalka et al. Our findings regarding autologous expanded EPC transplantation are consistent with those of Shintani et al, in which autologous expanded EPC from bone marrow promoted neovascularization in hind limb ischemia ${ }^{[22]}$. However, expanding EPC from peripheral blood is clearly less invasive than aspirating bone marrow. The culture-expanded EPC 
may consist of EPC at different stages of differentiation depending on the culture conditions or duration. Recently, Hur et al sequentially cultured total mononuclear cells from a source of adult peripheral blood and found 2 types of EPC: early and late EPC. However, there was no difference in the contributions of the 2 types to neovascularization in ischemic $\operatorname{limbs}^{[29]}$.

The incorporation rate of transplanted cells is greatly influenced by the delivery method. When infused intravenously, the number of infused cells incorporated in ischemic vasculature was much less than that being injected near the ischemic zone. To deliver the EPC efficiently, we directly injected the cells into the ischemic thigh muscle to ensure a maximum available concentration of EPC at the site of the ischemic hind limb. The optimal time for cell transplantation is also a primary concern; to mimic ischemic events in a clinical setting, and using the course of events after myocardial ischemia for reference, we set the time point for EPC injection at $\mathrm{d} 7$ after ischemia.

A limitation of the present study is that no control cells were used to demonstrate the advantage of expanded EPC. In a heterogenetic transplantation study with human EPC, Murasawa and Aahara used either human microvascular EC (HMVEC) or medium as a control in a nude mouse model of hind limb ischemia. They found that the outcomes in the mice receiving control cells (HMVEC) and those receiving the medium were similar ${ }^{[30]}$. The appropriate control cells for the present study would have been mature vascular EC. However, preparation of vascular EC for autologous transplantation is not feasible, so we had to use saline for the control.

In conclusion, we found that neovascularization was enhanced by autologous expanded EPC from peripheral blood when injected intramuscularly at the site of ischemia at $\mathrm{d} 7$ after hind limb ischemia in rabbits. Our data indicate that autologous expanded EPC from peripheral blood may be an important and novel therapeutic approach for ischemic disease via enhancing neovascularization.

\section{Acknowledgements}

The authors are very grateful to Prof Ke-min CHEN, Dr Jin-qing HU, and Dr Xian-wei ZENG from Ruijin Hospital, Shanghai, for their assistance in performing the angiography.

\section{References}

1 Risau W. Mechanisms of angiogenesis. Nature 1997; 386: 671-4.

2 Takeshita S, Zheng LP, Brogi E, Kearney M, Pu LQ, Bunting S, et al. Therapeutic angiogenesis. A single intraarterial bolus of vascular endothelial growth factor augments revascularization in a rabbit ischemic hind limb model. J Clin Invest 1994; 93: 66270 .

3 Harada K, Grossman W, Friedman M, Edelman ER, Prasad PV, Keighley CS, et al. Basic fibroblast growth factor improves myocardial function in chronically ischemic porcine hearts. J Clin Invest 1994; 94: 623-30.

4 Ylä-Herttuala S, Alitalo K. Gene transfer as a tool to induce therapeutic vascular growth. Nat Med 2003; 9: 694-701.

5 Baumgartner I, Pieczek A, Manor O, Blair R, Kearney M, Walsh $\mathrm{K}$, et al. Constitutive expression of phVEGF165 after intramuscular gene transfer promotes collateral vessel development in patients with critical limb ischemia. Circulation 1998; 97: 111423.

6 Shi Q, Rafii S, Wu MH, Wijelath ES, Yu C, Ihida A, et al. Evidence for circulating bone marrow-derived endothelial cells. Blood 1998; 92: 362-7.

7 Aahara T, Masuda H, Takahashi T, Kalka C, Pastore C, Silver M, et al. Bone marrow origin of endothelial progenitor cells responsible for postnatal vasculogenesis in physiological and pathological neovascularization. Circ Res 1999; 85: 221-8.

8 Iner JM, Aahara T. Angiogenesis and vasculogenesis as therapeutic strategies for postnatal neovascularization. J Clin Invest 1999; 103: 1231-6.

9 Takahashi T, Kalka C, Masuda H, Chen D, Silver M, Kearney M, et al. Ischemia- and cytokine-induced mobilization of bone marrow-derived endothelial progenitor cells for neovascularization. Nat Med 1999; 5: 434-8.

10 Aahara T, Murohara T, Sullivan A, Silver M, van der Zee R, Li T, et al. Isolation of putative progenitor endothelial cells for angiogenesis. Science 1997; 275: 964-7.

11 Kocher AA, Schuster MD, Szabolcs MJ, Takuma S, Burkhoff D, Wang $\mathrm{J}$, et al. Neovascularization of ischemic myocardium by human bone marrow-derived angioblasts prevents cardiomyocyte apoptosis, reduces remodeling and improves cardiac function. Nat Med 2001; 7: 430-6.

12 Kawamoto A, Gwon HC, Iwaguro H, Yamaguchi JI, Uchida S, Masuda $\mathrm{H}$, et al. Therapeutic potential of ex vivo expanded endothelial progenitor cells for myocardial ischemia. Circulation 2001; 103: 634-7.

13 Kalka C, Masuda H, Takahashi T, Kalka-Moll WM, Silver M, Kearney $\mathrm{M}$, et al. Transplantation of ex vivo expanded endothelial progenitor cells for therapeutic neovascularization. Proc Natl Acad Sci USA 2000; 97: 3422-7.

14 Kawamoto A, Tkebuchava T, Yamaguchi J, Nishimura H, Yoon YS, Milliken $\mathrm{C}$, et al. Intramyocardial transplantation of autologous endothelial progenitor cells for therapeutic neovascularization of myocardial ischemia. Circulation 2003; 107: 461-8

15 Tateishi-Yuyama E, Matsubara H, Murohara T, Ikeda U, Shintani $\mathrm{S}$, Masaki $\mathrm{H}$, et al. Therapeutic angiogenesis using cell transplantation (TACT) study investigators. Therapeutic angiogenesis for patients with limb ischemia by autologous transplantation of bone-marrow cells: a pilot study and a randomized controlled trial. Lancet 2002; 360: 427-35.

16 Asmus B, Schachinger V, Teupe C, Britten M, Lehmann R, Dobert $\mathrm{N}$, et al. Transplantation of progenitor cells and regeneration enhancement in acute myocardial infarction (TOPCARE-AMI). 
Circulation 2002; 106: 3009-17.

$17 \mathrm{Pu}$ LQ, Jackson S, Lachapelle KJ, Arekat Z, Graham AM, Lisbona $\mathrm{R}$, et al. A persistent hindlimb ischemia model in the rabbit. J Invest Surg 1994; 7: 49-60.

18 Fan CL, Li Y, Gao PJ, Liu JJ, Zhang XJ, Zhu DL. Differentiation of endothelial progenitor cells from human umbilical cord blood CD $34^{+}$cells in vitro. Acta Pharmacol Sin 2003; 24: 212-8.

19 Ito WD, Arras M, Scholz D, Winkler B, Htun P, Schaper W. Angiogenesis but not collateral growth is associated with ischemia after femoral artery occlusion. Am J Physiol 1997; 273: H125565.

20 Takeshita S, Rossow ST, Kearney M, Zheng LP, Bauters C, Bunting S, et al. Time course of increased cellular proliferation in collateral arteries after administration of vascular endothelial growth factor in a rabbit model of lower limb vascular insufficiency. Am J Pathol 1995; 147: 1649-60.

21 Flanagan MF, Fujii AM, Colan SD, Flanagan RG, Lock JE. Myocardial angiogenesis and coronary perfusion in left ventricular pressure-overload hypertrophy in the young lamb. Circ Res 1991; 68: $1458-70$.

22 Shintani S, Murohara T, Ikeda H, Ueno T, Sasaki K, Duan J, et al. Augmentation of postnatal neovascularization with autologous bone marrow transplantation. Circulation 2001; 103: 897-903.

23 Fuchs S, Baffour R, Zhou YF, Shou M, Pierre A, Tio FO, et al. Transendocardial delivery of autologous bone marrow enhances collateral perfusion and regional function in pigs with chronic experimental myocardial ischemia. J Am Coll Cardiol 2001; 37:
$1726-32$.

24 Strauer BE, Brehm M, Zeus T, Kostering M, Hernandez A, Sorg $\mathrm{RV}$, et al. Repair of infarcted myocardium by autologous intracoronary mononuclear bone marrow cell transplantation in humans. Circulation 2002; 106: 1913-8.

25 Perin EC, Dohmann HF, Borojevic R, Silva SA, Sousa AL, Mesquita $\mathrm{CT}$, et al. Transendocardial, autologous bone marrow cell transplantation for severe, chronic ischemic heart failure. Circulation 2003; 107: 2294-302.

26 Te HF, Kwong YL, Chan JK, Lo G, Ho CL, Lau CP. Angiogenesis in ischemic myocardium by intramyocardial autologous bone marrow mononuclear cell implantation. Lancet 2003; 361: 47-9.

27 Stamm C, Westphal B, Kleine HD, Petzsch M, Kittner C, Klinge $\mathrm{H}$, et al. Autologous bone-marrow stem-cell transplantation for myocardial regeneration. Lancet 2003; 361: 45-6.

28 Kang HJ, Kim HS, Zhang SY, Park KW, Cho HJ, Koo BK, et al. Effects of intracoronary infusion of peripheral blood stem-cells mobilized with granulocyte-colony stimulating factor on left ventricular systolic function and restenosis after coronary stenting in myocardial infarction: the MAGIC cell randomized clinical trial. Lancet 2004; 363: 751-6.

29 Hur J, Yoon CH, Kim HS, Choi JH, Kang HJ, Hwang KK, et al. Characterization of two types of endothelial progenitor cells and their different contribution to neovasculogenesis. Arterioscler Thromb Vasc Biol 2004; 24: 288-93.

30 Murasawa S, Aahara T. Endothelial progenitor cells for vasculogenesis. Physiology 2005; 20: 36-42. 